\title{
Un pequeño grupo de pacientes puede automonitorear su anticoagulación oral con warfarina en forma segura y efectiva
}

A small group of patients can auto-monitor their warfarin oral anticoagulation in an effective and safe way

Fitzmaurice D, et al. BMJ 2005; 331; 1057

\section{Objetivo}

Determinar en pacientes con anticoagulación oral (ACO) prolongada la efectividad del automonitoreo.

Diseño

Estudio multicéntrico abierto aleatorizado y controlado.

\section{Lugar}

Centros británicos de atención primaria (APS).

\section{Pacientes}

Fueron invitados 2470 mayores de 18 años recibiendo warfarina con indicación de AC mayor a 12 meses y aceptaron intervenir 617 pacientes (337 en el grupo intervención y 280 en el control) completando el seguimiento de 12 meses el $57 \%$.

\section{Intervención}

En fases 1 y 2 de estudio los pacientes fueron aleatorizados en una proporción de uno a uno, mientras que en fase 3 , esta fue de tres a dos a favor del grupo intervención. Los pacientes bajo intervención fueron entrenados para medirse la Razón Internacional Normatizada (RIN) dos veces por semana con un equipo de automonitoreo domiciliario (Coaguchek, Roche) y utilizar una tabla de corrección para modificar la dosis de warfarina.

\section{Medición de resultados principales}

Análisis por intención de tratar*. Porcentaje de tiempo en el que el RIN se mantuvo en el rango terapéutico.

\section{Resultados principales}

Se muestran en la tabla 1.
Tabla 1: porcentaje de tiempo transcurrido con anticoagulación en el rango adecuado en ambos grupos antes y después de la intervención.

\begin{tabular}{|c|c|c|c|c|}
\hline \multirow[b]{2}{*}{ Grupo } & \multicolumn{3}{|c|}{ Porcentaje de tiempo con RIN en rango } & \multirow[b]{2}{*}{ Cambio } \\
\hline & Previamente & a la intervención & $\begin{array}{l}\text { Durante la } \\
\text { intervención }\end{array}$ & \\
\hline \multirow[t]{2}{*}{ Auto-monitoreo } & $\begin{array}{l}\text { Buen control } \\
\quad(n=255)\end{array}$ & $74 \%(70,7$ a 77,2$)$ & $74 \%(72,3$ a 76,6$) \mid$ & $\begin{array}{c}\text { No } \\
\text { significativo }\end{array}$ \\
\hline & $\begin{array}{l}\text { Mal control } \\
\qquad(n=66)\end{array}$ & $45 \%(37,3$ a 51,7$)$ & $55 \%(50,0$ a 60,0$)$ & $\begin{array}{c}10,21 \\
(2,84 \text { a } 17,59)\end{array}$ \\
\hline \multirow[t]{2}{*}{ Control habitual } & $\begin{array}{c}\text { Buen control } \\
\quad(n=218)\end{array}$ & $72 \%(68,0$ a 75,3$)$ & $71 \%(67,8$ a 73,7$)$ & $\begin{array}{c}\text { No } \\
\text { Significativo }\end{array}$ \\
\hline & $\begin{array}{l}\text { Mal control } \\
\quad(n=38)\end{array}$ & $52 \%(42,2$ a 62,1$)$ & $53 \%(45,3$ a 60,0$)$ & \\
\hline
\end{tabular}

RIN: razón internacional normatizada.

Los pacientes en automonitoreo que previamente al estudio tenían mal control del RIN mostraron una mejoría que no fue vista en el grupo asignado a control habitual. Nueve pacientes (2,8/100 pacientes/año) tuvieron eventos adversos serios en el grupo de automonitoreo y siete (2,7/100 pacientes/año) en el grupo control habitual $(p=0,89)$. De los 337 aleatorizados a automonitoreo, $242(72 \%)$ completaron el entrenamiento y de los 242 que lo completaron, 193 (80\%) fueron seguidos por 12 meses; mientras que de los 280 aleatorizados a control habitual fueron seguidos 12 meses el 250 (89\%).

\section{Conclusiones}

Con entrenamiento adecuado el automonitoreo de la ACO puede mejorar el tiempo en que el RIN se mantiene en rango terapéutico en pacientes que previamente tenían mal control.

Palabras claves: anticoagulación oral, automonitoreo de anticoagulación, razón internacional normatizada.

Key words: oral anticoagulation, auto-monitoring, international normalized ratio.

Fuente de financiamiento/conflicto de interés de los autores: no referida.

\section{Comentario}

Cada vez mas pacientes requieren anticoagulación, por lo tanto se necesitan modelos terapéuticos alternativos ${ }^{1,2}$. Sin embargo, cualquier nuevo modelo de cuidado debe demostrar niveles de control superiores al $60 \%$ del tiempo en rango terapéutico, ser seguro y tener mas de un $10 \%$ de superioridad con respecto a los controles habituales ${ }^{3}$.

Este estudio evalúa la efectividad del automonitoreo de la ACO y la modificación de la dosis de warfarina con una tabla simple de corrección en un grupo de pacientes con indicación prolongada de ACO previamente entrenados; comparándola con el monitoreo realizado de la manera habitual con control de laboratorio y visita al profesional.

El automonitoreo de la ACO ha demostrado ser seguro y efectivo en otras poblaciones no comparables con Gran Bretaña.
Por ejemplo, en Alemania, el automonitoreo es rutinario y el $80 \%$ de los pacientes son capaces de realizarlo ${ }^{4}$, lo que probablemente sea reflejo del sistema de salud, de la motivación y de la capacidad de la población. Cabe aclarar que son menos de los esperados los pacientes que desean realizar el automonitoreo de la ACO.

\section{Conclusión de la comentadora}

El automonitoreo de la ACO oral demostró ser tan seguro y efectivo como el control de rutina en clínicas británicas de APS por lo menos para una minoría de pacientes apropiadamente entrenados y que ya estaban recibiendo warfarina.

María Paula Cárdenas [ Sección Hematología. Servicio de Clínica Médica.Hospital Italiano de Buenos Aires. ]

Cárdenas M. Evid. actual. práct. ambul; 10(4): 104, Jul-Ago.2007. Un pequeño grupo de pacientes puede automonitorear su anticoagulación oral con warfarina en forma segura y efectiva. Comentado de: Fitzmaurice D, et al, Self management or oral anticoagulation: randomised trial, BMJ 2005; 331; 1057. PMID: 16216821. Disponible en URL: http://www.bmj.com/cgi/reprint/331/7524/1057 (último acceso 31/07/07).

Referencias

1. Taylor F, Ramsey M, Voke J, Cohen H, GPs not prepaired for monitoring anticoagulation, BMJ 1993; 307:1493

1. Taylor F, Ramsey M, Voke J, Cohen H, GPs not prepaired for monitoring anticoagulation, BMJ 1993; 307:1493

2. Sudlow CM, Rodgers H, Kenny RA, Thompson RG, Service provision and use of anticoagulants in atrial fibrilation, BMJ 1995; 311:558-61
3. Fitzmaurice DA, Machin SJ, Recommendations for patients undertaking self management of oral anticoagulation, BMJ 2001;323:985-9

4. Morsdorf S, Erdlenbruch W, Taborski U, Schenk JF, Erdlenbruch K, Novotny- Reichert G, et al, Training of patients for self-management or oral anticoagulant therapy: standards, patient suitability and clinical aspects, Semin Thromb Hemost 1999;25:109-15 